\title{
Evaluation of Delirium Course Program Given to Intensive Care Nurses
}

\author{
Gamze Sarikoc $^{1^{*}}$ and Ozlem Canbolat ${ }^{2}$ \\ ${ }^{1}$ Department of Mental Health and Psychiatric Nursing, Gulhane Faculty of Nursing, Health Sciences University, \\ Ankara, Turkey \\ ${ }^{2}$ Department of Internal Medicine Nursing, Faculty of Nursing, Necmettin Erbakan University, Konya, Turkey
}

*Corresponding author: Gamze Sarikoc, Ph.D., Assist. Prof., Department of Mental Health and Psychiatric Nursing, Gulhane Faculty of Nursing, University of Health Sciences TURKEY, Ankara, Turkey, Tel: +90-053-064-22-313, Fax: +90312-304-39-70

\begin{abstract}
Background: Delirium is frequently seen in intensive care units. Because of the negative consequences and increased healthcare costs associated with delirium, prevention interventions are crucial to reducing the frequency and complications of delirium. The most basic component in the prevention of delirium is that the patient should be evaluated in terms of delirium risk factors within the first 24 hours of admission to the clinic. Raising awareness about delirium and increasing the level of knowledge of nurses in this subject is important in terms of pre-determination of delirium and ensuring patient safety.
\end{abstract}

Objective: This study examined that evaluation of a delirium course program for ICU nurses delivered by using standardized patients (SPs).

Method: This research was organized as qualitative. This research was conducted with a sample of 22 intensive care nurses by delirium course program which was three steps given to nurses by using SP. SP was evaluated by nursing using the Turkish version of the intensive care delirium screening checklist followed by a semi-structured interview of participants and qualitative analysis of responses. Nurses participated in the study on a voluntary basis.

Results: Following the delirium course program nurses stated that their self-esteem towards the approach of the patient in delirium increased and can control their emotions after training and learn that their patients should not personalize their behavior.

Conclusion: Delirium course program with SPs increased nurses' self-esteem about delirium management. It also changed their perceptions of and attitudes toward patients positively. It is recommended to conduct studies evaluating whether the training provided reflects on the clinical practice of nurses.

\section{Keywords}

Delirium, Nursing, Training

\section{Introduction}

Delirium develops in short time and has a fluctuating course. It is the deterioration of retrospective mental state categorized as attention (attention orientation, focusing and shifting focus) and cognitive change [1]. According to the Diagnostic and Statistical Manual of Mental Disorders (DSM 5) delirium is defined disturbance inattention, awareness and cognition with reduced ability to direct, focus, sustains and reduced orientation to the environment [1]. Delirium is characterized by sudden disturbances in consciousness, attention, perception, sleep, psychomotor activity and emotion that fluctuate throughout the day, usually developing in a short time such as hours or days, and can last for weeks or months $[2,3]$.

The prevalence of delirium in intensive care patients ranges from 11 to $75.6 \%$ [4-7], and $62.5 \%$ of patients in mechanical ventilator [6]. Delirium can lead to prolonged of hospitalization and staying in intensive care unit, increase mortality and morbidity $[8,9]$. Although patients with delirium are described as hyperactive in current epidemiological evidence [10], it was found that hypoactive and mixed delirium are high in patients treated in intensive care units [11]. In hyperactive delirium catheter withdrawal and extubating may occur, and in hypoactive patients with changes in cognitive 
functions that are ignored delirium is often disregarded and this increases their aspiration and reintubation risk $[5,12]$. Major problems with patient safety and care occur due to these factors.

The Clinical Practice Guidelines for Pain, Agitation/ Sedation, Delirium Prevention and Management (PAD) and the National Institute for Health and Care Excellence (NICE) for delirium mentions evidence-based practices and results. In these guidelines, risk factors that are effective for the onset of delirium are mentioned $[8,13]$. The risk factors that affect delirium in intensive care units are dementia, coma, pre-intensive surgery or trauma, high APACHE II score, high ASA score, hypertension, a neurological disease, respiratory diseases, persistent venous hemofiltration and low Glasgow Coma Score [13].

Determine delirium risk factors and the evidence-level results presented are important. In order to prevent delirium, patients will be protected from delirium and its complications, thanks to the necessary interventions for risk factors that can be prevented. In fact, up to a third of delirium attacks can be prevented [8]. The most basic component in the prevention of delirium is the detection and control of predisposing and precipitant factors. The patient should be evaluated for delirium risk factors by the healthcare personnel within the first 24 hours of admission to the clinic [13].

In this context, health professionals working in clinical care should be educated about delirium and risk factors and protocols should be developed in this direction. There is an example of a protocol named HELP (Hospital Elder Life Program) in the literature. In this intervention, patients were followed with a standardized protocol for six risk factors (cognitive impairment, sleep deprivation, inactivity, visual impairment, hearing impairment, and dehydration). The effectiveness of this intervention reduced the incidence of delirium in $40 \%$ of cases, and delirium attacks resulted in fewer days [14]. Modified HELP was applied to elderly patients who underwent abdominal surgery, and it was reported that the protocol reduced delirium rates by $56 \%$ and hospitalization days by 2 days [15].

Therefore, it is important to determine, observe and implement nursing interventions that diagnose the symptoms of delirium early. In PAD's recommendations for pain, agitation and delirium for critical care patients, it is necessary to continuously evaluate delirium with scales for the prevention of delirium at the level of Evidence $A$ [8]. Validity and reliability assessment tools are available to enable patients to be evaluated in terms of delirium. The gold standard for delirium detection, the CAM-confusion assessment method is one of them [16]. The most valid and reliable scales developed to evaluate patients in intensive care units. One of them is Intensive Care Delirium Screening Checklist-(ICDSC) [17,18].
However, in one study, they stated that they could not evaluate because of the difficulty of using the evaluation tools, it was not easy to evaluate the patients who were intubated or sedated, because nurses were hesitant to use the evaluation tools and the belief that using the evaluation tools would not affect the patient results [19]. According to the worldwide study of Morandi, et al. to evaluate the use of the care packet, which they named as ABCDEF (Assessing Pain, Both Spontaneous Awakening and Breathing Trials, Choice of Drugs, Delirium Monitoring, Early Exercise and Family Empowerment), delirium monitoring rate in ICUs is $70 \%$ but it was found that only $42 \%$ used an assessment tool for delirium [20]. According to study by Devlin and et al., half of intensive care nurses stated that they could not do delirium assessment in their units because the scales were complicated and their patients had intubation [21].

The nurses did not have sufficient knowledge and therefore were unable to recognize patients with delirium $[22,23]$. A study assessing the delirium knowledge and skill levels of intensive care nurses in Jordan found that they lacked sufficient knowledge and skills [24]. A study conducted in Egypt assessed the awareness and nursing intervention of 120 intensive care nurses' for delirium. It found that more than half the nurses (54.2\%) did not assess delirium and none of them had been trained to assess and treat delirium [25]. A study by Voyer, et al. conducted with 156 nurses found that only $13 \%$ of them had diagnosed cases of delirium. 16 another studies have been shown that nurses think delirium as important and serious health problems at patients in the intensive care units, but in delirium management and taking the necessary measures have been shown to be insufficient $[26,27]$.

Delirium is frequently seen in intensive care units and associated with increased morbidity and mortality, cost of care, complications from hospital, slower physical recovery rate, poor functional and cognitive recovery, low quality of life $[2,9,28]$. So delirium is a serious condition that needs early diagnosis and treatment in intensive care units. The most basic component in the prevention of delirium is to detect and control the predisposing and precipitant factors [13]. Nurses need sufficient knowledge, skill, and experience to play an active role in assessing and managing delirium. To this end, e-learning, delirium training or interdisciplinary courses are beneficial for nurses to make early diagnoses and learn necessary interventions and practices $[29,30]$. One of these interactive training methods is standardized patient practice. The standardized patient (SP) practice described by Barrows in 1963 can be used to improve health care staff's knowledge and skills today [31]. Barrows described as "SP is a person who has been carefully coached to simulate an actual patient so accurately that simulation cannot be detected by a skilled clinician." [32]. SP training includes acting out scenarios such as patients having difficulty with communication, difficult 
patients and end-of-life care and enable the students to enjoy during these post-graduation training [33-35]. Haugland and Reime applied scenario-based simulation in dementia care training to nursing students. They stated that simulation training seems to be a useful approach for preparing students to become more aware of demanding situations in dementia care [36]. Witt, et al. examined whether engaging SPs in a simulated home environment enhanced nursing students' competency in assessment and communication with patients with mental health conditions. It was stated that SP practices can be an effective strategy to prepare nursing students for mental health clinical experiences [37]. It is thought that raising awareness about delirium and increasing the level of knowledge of nurses in this subject is important in terms of pre-determination of delirium and ensuring patient safety. Therefore, the aim of this study is to evaluate the delirium course program supported by the SP given to the intensive care nurses.

\section{Methods}

\section{The aim of the study}

This research study was conducted to evaluate the delirium course program supported by the SP given to the intensive care nurses. This research was organized as qualitative.

\section{Population and sampling}

The data of this study, which evaluated the delirium course program given to intensive care nurses, were collected by semi-structured focus group interviews between Feb-May 2017. The nurses who participated to study were working in intensive care unit of a hospital in Turkey. This intensive care unit is 11 bed capacity and provides third level intensive care service. No sample selection was made, this study conducted with 22 nurses who wanted to participate. Nurses participated in the study on a voluntary basis. Participants were informed about the course program and the study and were informed that they had the right to participate in the survey and that they could leave without study at any time.

\section{Procedure}

When the implementation of the course program and the implementation of the data collection tools are fully addressed, the implementation process is three steps. The course program is a short-term (half-day) face to face initiative for every 3-4 nurses. In this direction;

Self-identification: This section assessed years of professional experience, education levels and encounters with patients with delirium and training about delirium. These questions were asked before the course program to help recognize the characteristics of the group.

Curriculum: In this step, training on delirium was given to the nurses by the researchers. This course program includes information about delirium diagnostic criteria, delirium types, risk factors, etiology, evaluation methods and nursing interventions. Then, the nurses evaluated the SP who enrolled patient with delirium. The nurses had the opportunity to implement appropriate interventions with the patient who portrayed the delirium case. Standardized patient is selected from people who have a role-playing experience, who previously worked as a patient in the training of health professionals, and who have the potential to make the scenario relevant to reality. The SP was trained to portray the patient with delirium prior to the course program. The patient with delirium is enrolled by the SP in a room created by intensive care conditions. Nurses were asked to use the "Intensive Care Delirium Screening Checklist" to assess the delirium type of (hyperactive type) patient and plan appropriate interventions. This scale, which is validated by Köse, et al. in Turkish, is completed by the nurses with information obtained from the 24-hour or 8-hour shift. This scale consists of eight parts that evaluate the patient. The scale total score is obtained as the result of scoring given to each part (See Appendix 1) $[17,18]$. Necessary permissions have been obtained from the authors to use the checklist. This scale was used not as a measurement tool in the study but as an example tool for the nurses for assessing the patient with delirium and for evaluating the delirium by an objective scoring system. Following this interview with the patient, the SP gave feedback to the nurses about the effects of the interventions made by them. Then, debriefing sessions were held.

Post-training assessment: Semi-structured, faceto-face interviews were conducted with groups of 3-4 nurses to learn the evaluations of nurses about the course program by researchers. As soon as the course program was finished, interviews were conducted. Semi-structured interviews were held in a special room that would not dissuade the group's attention, which would not affect the work flow of the nurses. In these interviews, nurses were directed to the following questions; evaluation the course program, course programs' effect on nurses perceptions and attitudes towards the patient with delirium, course programs' effect on nurses skills in approaching the patient with delirium, suggestions and contributions about the course program. Six focus group interviews were conducted with 22 nurses. The responses of the participants were recorded with a voice recorder and written by listening. Participants were informed that the interviews were recorded. Transcripts are examined independently and similar, repetitive expressions are encoded. Participants are coded by numbering instead of their names. Then, these encodings were categorized and the themes were created. While themes were creating, the opinions of two academicians who are qualified in the field of qualitative research were also taken. Academics independently coded the answers. Then, the answers were given ac- 
cording to the themes according to the density ratio.

\section{Ethical considerations}

Ethics committee approval was obtained from health care organization's ethics committee (02-2017/1344). The study was conducted in accordance with the principles of the Declaration of Helsinki. Each participant was informed about the study process and written approval was obtained.

\section{Results}

All the nurses were female and had bachelor's degrees. Of the nurses, $31.8 \%$ had worked for less than a year, $45.4 \%$ had worked for 2 to 5 years, and $22.7 \%$ had worked for 6 to 9 years. Of them, $50 \%$ had not received training about delirium, and $81.8 \%$ had encountered patients with delirium.

\section{Semi-structured interviews}

Based on the findings obtained, "Self-confidence", "Perception and attitudes towards illness" and "Satisfaction with course program" were identified as themes.

\section{$1^{\text {st }}$ theme: Self-confidence}

The participants stated that they felt better about how to approach the patient with delirium.

"It's a frequently encountered situation. It was very beneficial to learn that these symptoms are symptoms of delirium and realize their importance", "It raised my awareness. I did not know that I should inform doctors", "I am now more poised and aware if I encounter such a patient", "I was unsure about how to approach these patients before. Now I can approach them more systematically and professionally", "I trusted myself about this subject, but I saw that I did not have enough information... I think that I have refreshed my knowledge and improved", "Sometimes we ignore things in work life. I did not know that delirium is an emergent medical situation. This training raised our awareness", "It seemed normal to me at first, but then I learned that I cannot handle this by myself, and I need to inform doctors".

\section{$2^{\text {nd }}$ theme: Perception and attitudes towards illness}

Participants of the training had the opportunity to review their own feelings and behaviors towards the patient with delirium. They stated that the negative feelings about the next behaviors decreased even more.

"I was blaming myself. I will try not to take what patients do personally and get angry", "I realized that patients do things for a reason, and they are not about me", "We deal with many patients, and this was ordinary to us. Now I realize that it is an emergency", "I can be more patient".

\section{$3^{\text {rd }}$ theme: Satisfaction with course program}

Participants stated that the inclusion of the application in the training program is effective and makes the training interesting.

"It was different, fun and catchy", "You do not see every symptom in every patient. We had chance to see many symptoms and findings thanks to standardized patients", "It was nice and informative, and then practicing increased its permanence", "It was good to apply the scale to standardized patients. I understand it better this way. I saw that I can do it when I try", "Using scales with standardized patients helped us to do this", "We would not have completely understood if we did not have standardized patient practice", "It was enjoyable training".

\section{Discussion}

Most of the nurses who participated in program found it enjoyable and instructive. Members of professions that provide services for humans such as health care staff need to improve and develop their skills. Adults like to learn in comfortable, safe, and dynamic training environments [38]. The nurses in our study interacted with standardized patients after theoretical training. They assessed the patients using a scale and planned suitable nursing interventions. The nurses assessed themselves in a safe environment and discussed the effects of their interventions in feedback and debriefing sessions. A study that assessed satisfaction with using standardized patients in psychiatric nursing training to teach critical thinking skills and self-esteem found that its students were very satisfied with critical thinking, self-esteem and the training [39]. Bowers, et al. assessed the effect of standardized patient practice about insulin injection and counseling on freshman nursing students. Their study found that standardized patient practice effectively increased the students' knowledge and skill levels [40].

The nurses stated that they feel guilty when they encounter patients with delirium and sometimes, they got angry because they took patients' behaviors personally. The training taught them that they can control their emotions, and that they should not take patients' behaviors personally. A study by Detroyer, et al. examined the effect of an e-learning delirium tool for delirium recognition on knowledge and the stress of caring for patients with delirium. The health care staff's delirium recognition and knowledge levels increased, but their stress did not change [30]. A qualitative study conducted with nurses caring for patients with delirium in intensive care units found that they experienced physical and psychological stress and had dilemmas about making decisions [41]. Nurses who observe and assess patients for 24 hours have frequent interactions with patients. Therefore, nurses can play an active role in providing physiological, psychological, and environmental support and symptomatic care [42]. Hyperactive delirium patients pulling bed sheets, yelling, swearing, and trying to pull off tubes and drains were observed. These situations make nurses get angry, have negative emo- 
tions toward patients, and feel exhausted and desperate [43]. Training programs explaining these symptoms in patients and reasons for them will increase nurses' knowledge levels. Thus, nurses' negative experiences can be reduced, and their will to help can increase. Real patients as a training resource are used for physical examinations, medical history, skill training and different training environments [44]. In addition, using SPs offers the opportunity to redo scenarios whenever wanted and the necessary number of times [45]. Previous studies have shown that training conducted with standardized patients is enjoyable, satisfying and educational $[46,47]$. A training environment where learners participate actively, are happy and satisfied and can boost their motivation can make learning permanent.

Intensive care unit nurses spend a lot of time in intensive care with patients and try to improve their skills all the time. They realize that recognizing problems at an early stage is the key point of early diagnosing delirium in patients. Previous studies of delirium diagnoses found that nurses did not have enough knowledge and were thus unable to recognize patients with delirium $[22,23]$. Giving nurses undergraduate training about delirium effectively helps them to diagnoe delirium at an early stage [48]. The nurses who were trained stated that this training raised their self-confidence. A study by Akechi, et al. carried out with 390 nurses researched the effect of a delirium course program on the self-esteem of nurses regarding giving care to patients with delirium. It found that nurses' self-esteem increased in distinguishing delirium from dementia, psychosis and depression [49]. A pretest and posttest model was used to assess the effectiveness of an e-learning course regarding delirium for nurses working in internal disease and surgical clinics in 17 hospitals in Holland. The course was intended to raise their awareness about delirium, related factors and nursing interventions. The training increased the nurses' knowledge levels about nursing interventions and delirium [29]. Milisen, et al. assessed the effect of an interdisciplinary program about delirium on delirium development frequency, cognitive functions, functional rehabilitation level, morality and hospitalization length in elderly patients with hip fractures and found that it had positive effects on early diagnosis and treatment of delirium [50]. In our day, knowledge needs to be updated quickly according to the needs of a changing era. Updating current information and reminding forgotten knowledge through a program are considered to help nurses. Nurses whose knowledge and skill levels are increased have increased self-confidence, which positive effects on care quality and motivation. Practical measurement tools for assessing delirium can help to manage it. The Intensive Care Delirium Screening Checklist used for the assessment of patients with delirium gave the nurses the opportunity to practice the knowledge they learned with SPs.

\section{Conclusion and Recommendations}

Thanks to our course program, the nurses' self-confidence increased, positive changes in perceptions of and attitudes toward patients with delirium were observed, and a measurement tool was used for delirium management. Delirium management made a difference in the nurses' treatment of critical patients. More studies and training are needed for delirium management in nursing. Different training techniques and studies are recommended to support an active role of nurses in delirium management. In addition, it is recommended to conduct studies evaluating whether the training provided reflects on the clinical practice of nurses.

\section{Study Limitations}

The course program is a short-term (half-day) initiative. For both nurses, the intensity of the working environment, and the excess work load, made it difficult to plan a long course program. It is thought that the results of the study, which are compared by using a combination of longer and different interactive education methods, may be more effective. The fact that the study is single-centered, and the low number of samples is another limitation. The results of this research can only be generalized to this sample group.

\section{Acknowledgement}

No financial assistance was required for this research study.

\section{Conflict of Interest}

None.

\section{References}

1. American Psychiatric Association. Diagnostic and statistical manual of mental disorders (DSM-5).

2. Tieges Z, Evans JJ, Neufeld KJ, MacLullich AMJ (2018) The neuropsychology of delirium: Advancing the science of delirium assessment. Int J Geriatr Psychiatry 33: 15011511.

3. Johansson YA, Bergh I, Ericsson I, Sarenmalm EK (2018) Delirium in older hospitalized patients-signs and actions: A retrospective patient record review. BMC Geriatr 18: 43.

4. Salluh JIF, Wang H, Schneider EB, Nagaraja N, Yenokyan G, et al. (2015) Outcome of delirium in critically ill patients: Systematic review and meta-analysis. BMJ 350: 2538.

5. Lin SM, Liu CY, Wang CH, Lin HC, Huang CD, et al. (2004) The impact of delirium on the survival of mechanically ventilated patients. Crit Care Med 32: 2254-2259.

6. Limpawattana P, Panitchote A, Tangvoraphonkchai K, Suebsoh N, Eamma W, et al. (2016) Delirium in critical care: A study of incidence, prevalence, and associated factors in the tertiary care hospital of older Thai adults. Aging Ment Health 20: 74-80.

7. Heriot NR, Levinson MR, Mills AC, Khine TT, Gellie AL, et al. (2017) Diagnosing delirium in very elderly intensive care patients. Intensive Crit Care Nurs 38: 10-17.

8. Devlin JW, Skrobik Y, Gélinas C, Needham DM, Slooter 
AJC, et al. (2018) Clinical practice guidelines for the prevention and management of pain, agitation/sedation, delirium, immobility, and sleep disruption in adult patients in the ICU. Crit Care Med 46: e825-e873.

9. Schubert M, Schürch R, Boettger S, Nuñez DG, Schwarz $U$, et al. (2018) A hospital-wide evaluation of delirium prevalence and outcomes in acute care patients - A cohort study. BMC Health Serv Res 18: 550.

10. Kim H, Chung S, Joo YH, Lee JS (2016) The major risk factors for delirium in a clinical setting. Neuropsychiatr Dis Treat 12: 1787-1793.

11. Krewulak KD, Stelfox HT, Leigh JP, Ely EW, Fiest KM (2018) Incidence and prevalence of delirium subtypes in an adult ICU: A systematic review and meta-analysis. Crit Care Med 46: 2029-2035.

12. Dubois MJ, Bergeron N, Dumont M, Dial S, Skrobik $Y$ (2001) Delirium in an intensive care unit: A study of risk factors. Intensive Care Med 27: 1297-1304.

13. NICE guideline CG103 (2010) Appendix A: Summary of evidence from surveillance. 2018 surveillance of Delirium: Prevention, diagnosis and management.

14. Inouye SK, Baker DI, Fugal P, Bradley EH, HELP Dissemination Project (2006) Dissemination of the hospital elder life program: Implementation, adaptation, and successes. J Am Geriatr Soc 54: 1492-1499.

15. Chen CC, Li HC, Liang JT, Lai IR, Purnomo JDT, et al. (2017) Effect of a modified hospital elder life program on delirium and length of hospital stay in patients undergoing abdominal surgery: A cluster randomized clinical trial. JAMA Surg 152: 827-834.

16. Inouye SK, van Dyck CH, Alessi CA, Balkin S, Siegal AP, et al. (1990) Clarifying confusion: The confusion assessment method. A new method for detection of delirium. Ann Intern Med 113: 941-948.

17. Kose G, Bolu A, Ozdemir L, Acikel C, Hatipoglu S (2016) Reliability and validity of the intensive care delirium screening checklist in Turkish. Int J Nurs Knowl 27: 119-124.

18. Bergeron N, Dubois MJ, Dumont M, Dial S, Skrobik Y (2001) Intensive care delirium screening checklist: Evaluation of a new screening tool. Intensive Care Med 27: 859-864.

19. Awad SA (2019) Critical care nurses' knowledge, perception and barriers regarding delirium in adult critical care units. American Journal of Nursing Research 7: 193-198.

20. Morandi A, Piva S, Ely EW, Myatra SN, Salluh JIF, et al. (2017) Worldwide survey of the "Assessing pain, both spontaneous awakening and breathing trials, choice of drugs, delirium monitoring/management, early exercise/ mobility, and family empowerment" (ABCDEF) bundle. Crit Care Med 45: e1111-e1122.

21. Devlin JW, Fong JJ, Howard EP, Skrobik Y, McCoy N, et al. (2008) Assessment of delirium in the intensive care unit: Nursing practices and perceptions. Am J Crit Care 17: 555565 .

22. Voyer P, Richard S, Doucet L, Danjou C, Carmichael PH (2008) Detection of delirium by nurses among long-term care residents with dementia. BMC Nurs 7: 4.

23. Rice KL, Bennett M, Gomez M, Theall KP, Knight M, et al (2011) Nurses' recognition of delirium in the hospitalized older adult. Clin Nurse Spec 25: 299-311.

24. Hamdan-Mansour AM, Farhan NA, Othman EH, Yacoub Mi (2010) Knowledge and nursing practice of critical care nurses caring for patients with delirium in intensive care units in
Jordan. J Contin Educ Nurs 41: 571-576.

25. Elfeky HA, Ali FS (2013) Nurses' practices and perception of delirium in the intensive care units of a selected university hospitals in Egypt. Journal of Education and Practice 4: 61-71.

26. Zeki O (2013) Thoughts and attitudes of nurses about the management of delirium. Postgraduate Thesis, Psychiatric Nursing ABD, Institute of Health Science, Istanbul University.

27. Cevik B, Akyuz E, Ugurlu Z, Ersayın A, Dogan N (2016) Determination of the awareness about delirium management of nurses working in intensive care units. Capital University Health Sciences Journal 1: 32-46.

28. Maldonado JR (2015) Delirium: Neurobiology, characteristics and management. In: Fogel BS, Greenberg DB, Psychiatric care of the medical patient. ( $3^{\text {rd }}$ edn), Oxford University Press, New York, NY, 823-907.

29. Steeg L, ljkema R, Wagner C, Langelaan M (2015) The effect of an e-learning course on nursing staff's knowledge of delirium: A before and after study. BMC Med Educ 15: 12.

30. Detroyer E, Dobbels F, Debonnaire D, Irving K, Teodorczuk A, et al. (2016) The effect of an interactive delirium e-learning tool on healthcare workers' delirium recognition, knowledge and strain in caring for delirious patients: A pilot pretest/post-test study. BMC Med Educ 16: 17.

31. Schlegel C, Bonvin R, Rethans JJ, Van der Vleuten C (2016) Standardized patients' perspectives on workplace satisfaction and work-related relationships: A multicenter study. Simul Healthc 11: 278-285.

32. Barrows HS (1987) Simulated (Standardized) patients and other human simulations. Health Sciences Consortium 1, Chapel Hill.

33. Marken PA, Zimmerman C, Kennedy C, Schremmer R, Smith KV (2010) Human simulators and standardized patients to teach difficult conversations to interprofessional health care teams. Am J Pharm Educ 74: 120.

34. Jennings A (2017) Utilizing standardized patients to teach motivational interviewing to gerontology health care Providers. J Gerontol Geriatr Res 6: 1-2.

35. Fink M, Linnard-Palmer L, Ganley B, Catolico O, Phillips W (2014) Evaluating the use of standardized patients in teaching spiritual care at the end of life. Clinical Simulation in Nursing 10: 559-566.

36. Haugland VL, Reime MH (2018) Scenario-based simulation training as a method to increase nursing students' competence in demanding situations in dementia care. A mixed method study. Nurse Educ Pract 33: 164-171.

37. Witt MA, McGaughan K, Smaldone A (2018) Standardized patient simulation experiences improves mental health assessment and communication. Clinical Simulation in Nursing 23: 16-20.

38. Hacialioglu $N$ (2011) Teaching, learning and education in nursing. Nobel Medical Bookstores, 33-39, 103-110.

39. Robinson-Smith G, Bradley P, Meakim C (2009) Evaluating the use of standardized patients in undergraduate psychiatric nursing experiences. Clinical Simulation in Nursing 5: 203-211.

40. Bowers R, Tunney R, Kelly K, Mills B, Trotta K, et al. (2017) Impact of standardized simulated patients on first-year pharmacy students' knowledge retention of insulin injection technique and counseling skills. Am J Pharm Educ 81: 113. 
41. Yue $P$, Wang L, Liu C, Wu Y (2015) A qualitative study on experience of nurses caring for patients with delirium in ICUs in China: Barriers, burdens and decision making dilemmas. International Journal of Nursing Sciences 2: 2-8.

42. Boot $R$ (2012) Delirium: A review of the nurses' role in the intensive care unit. Intensive Crit Care Nurs 28: 185-189.

43. Oksuz E, Mersin S (2014) Confused patient. In: Oz F, Demiralp M, Psychosocial nursing for general patient care. Academician Medical Bookstore, 157-177.

44. Bokken L, Rethans JJ, Scherpbier AJ, van der Vleuten CP (2008) Strengths and weaknesses of simulated and real patients in the teaching of skills to medical students: A review. Simul Healthc 3: 161-169.

45. Cleland JA, Abe K, Rethans JJ (2009) The use of simulated patients in medical education: AMEE Guide No 42. Med Teach 31: 477-486.
46. Anderson M, Holmes TL, LeFlore JL, Nelson KA, Jenkins T (2010) Standardized patients in educating student nurses: One school's experience. Clinical Simulation in Nursing 6: E61-E66.

47. Luctkar-Flude M, Wilson-Keates B, Larocque M (2012) Evaluating high-fidelity human simulators and standardized patients in an undergraduate nursing health assessment course. Nurse Educ Today 32: 448-452.

48. Tabet N, Hudson S, Sweeney V, Sauer J, Bryant C, et al. (2005) An educational intervention can prevent delirium on acute medical wards. Age Ageing 34: 152-156.

49. Akechi T, Ishiguro C, Okuyama T, Endo C, Sagawa R, et al. (2010) Delirium training program for nurses. Psychosomatics 51: 106-111.

50. Milisen K, Foreman MD, Abraham IL, Geest SD, Godderis J, et al. (2001) A nurse-led interdisciplinary intervention program for delirium in elderly hip-fracture patients. J Am Geriatr Soc 49: 523-532. 
Appendix 1: Intensive care delirium screening checklist.

\begin{tabular}{|l|l|l|l|l|}
\hline Patient Evaluation & Day 1 & Day 2 & Day 3 & Day 4 \\
\hline Altered level of consciousness* (A-E) & & & Day 5 \\
\hline If A or B do not complete patient evaluation for the period & & & \\
\hline Inattention & & & \\
\hline Disoritentation & & & \\
\hline Hallucination-Delusion-psychosis & & & \\
\hline Psychomotor agitation or retardation & & & \\
\hline Inappropriate speech or mood & & & \\
\hline Sleep/wake cycle disturbance & & & \\
\hline Symptom fluctuation & & & \\
\hline Total Score (0-8) & & & \\
\hline
\end{tabular}

"Level of consciousness:

A: No response, score: None

B: Response to intense and repeated stimulation (loud voice and pain), score: None

C: Response to mild or moderate stimulation, score: 1

D: Normal wakefullness, score: 0

E: Exaggerated response to normal stimulation, score: 1 